\title{
Bibliothek vonRoll und Bibliothek Münstergasse: zwei Standortschwerpunkte im dezentral aufgebauten Bibliothekssystem der Universitätsbibliothek Bern
}

\begin{abstract}
Die Universitätsbibliothek Bern (UB Bern) konnte in den ersten zehn Jahren ihres Bestehens im Hochschulzentrum vonRoll ein grosses Neubau- und an der Münstergasse ein umfangreiches Umbauprojekt realisieren. Beides waren ,Generationenprojekte', die nur alle paar Jahrzehnte anstehen. Die Projekte haben es ermöglicht, Bestände und einzelne Prozesse $\mathrm{zu}$ zentralisieren sowie Dienstleistungen zu überdenken. Dadurch sind an den zwei grössten Standorten der UB Bern attraktive Räume mit guter Infrastruktur für Studierende und Mitarbeitende entstanden. Dahinter steht das Konzept der Standortschwerpunkte in einem gewachsenen und dezentral aufgebauten universitären Bibliotheksnetz. Das Zusammenführen von kleinen Teilbibliotheken zu grösseren Einheiten wird auch in Zukunft eine strategisch wichtige Aufgabe der UB Bern bleiben.
\end{abstract}

\section{Universitäre Bibliotheken in Bern}

Als die Universitätsbibliothek Bern 2007 gegründet wurde, zählte sie 49 Teilbibliotheken an 30 verschiedenen Adressen. Seither hat sich diese Zahl durch mehrere Reorganisationen und den Bau der Bibliothek vonRoll auf 39 Teilbibliotheken reduziert. Die UB Bern ist damit immer noch sehr dezentral organisiert.

Diese räumliche und organisatorische Struktur erklärt sich aus der Geschichte der Universität. Am ersten Standort der Hochschule in der Altstadt existierte seit der Reformationszeit vor 500 Jahren eine Bibliothek der Theologischen Hochschule. Ende des 19. Jahrhunderts und Jahrzehnte nach der Gründung der modernen Universität 1834 begann sich die Universität von der Altstadt in das Länggassquartier zu verlagern. Dort siedelten sich zuerst neue Institute an, die bis um 1900 knapp 20 eigene Bibliotheken aufbauten. 1903 wurde das neue Hauptgebäude der Universität auf der Grossen Schanze oberhalb des Bahnhofes zum neuen Schwerpunkt der höheren Bildung. An der Münstergasse in der Altstadt verblieb schliesslich nur die Stadt- und Universitätsbibliothek (StUB), die bezüglich ihrer Bestände und des Personals bis 2013 der grösste Standort des

Ә Open Access. (C) Christian Lüthi, publiziert von De Gruyter. (c) BY-NC-ND Dieses Werk ist lizenziert unter der Creative Commons Attribution-NonCommercial-NoDerivatives 4.0 Lizenz. https://doi.org/10.1515/9783110553796-024 
universitären Bibliothekswesens in Bern und bis 2007 aber nicht Teil der Universität war. $^{1}$

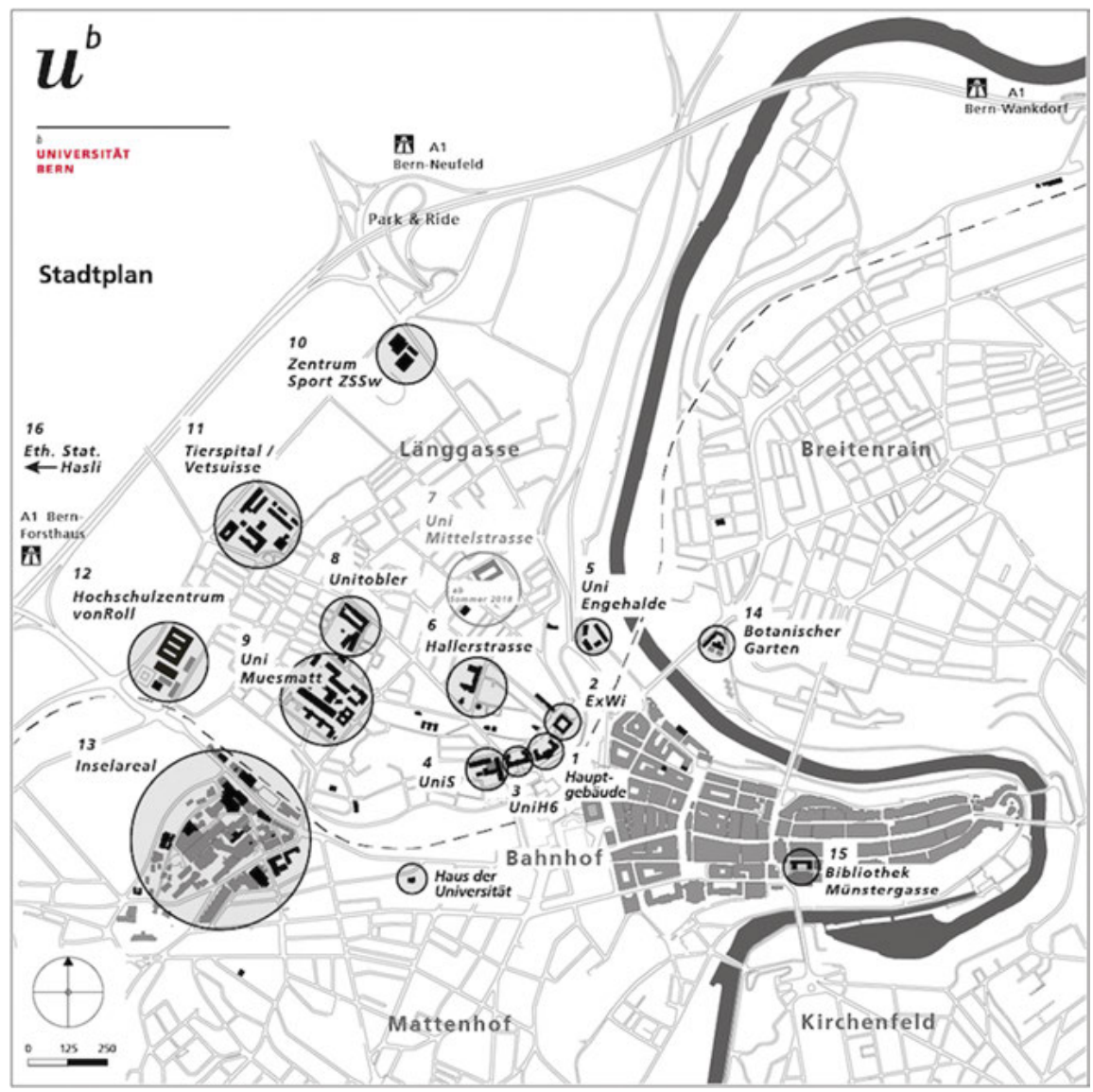

Abb. 1: Standorte der Universität Bern in der Stadt Bern (@ Universität Bern, Abteilung Bau und Raum)

1 Hans A. Michel: Das wissenschaftliche Bibliothekswesen Berns vom Mittelalter bis zur Gegenwart. In: Berner Zeitschrift für Geschichte und Heimatkunde 47 (1985), S. 267-234, hier S. 208f., 216-228 (http://doi.org/10.5169/seals-246337); Pietro Scandola (Red.): Hochschulgeschichte Berns 1528-1984. Zur 150-Jahr-Feier der Universität Bern 1984. Bern 1984, S. 588f., 769-776; Anna Minta, Bernd Nicolai, Markus Thome (Hrsg.): Stadt Universität Bern. 175 Jahre Bauten und Kunstwerke. Bern u.a.. 2009, S. 130-133, 157-165, 205-209, darin: Kilian Bühlmann: Bauen für die Universität, S. 115-127; Robert Barth, Emil Erne, Christian Lüthi (Hrsg.): Bern - die Geschichte der Stadt im 19. und 20. Jahrhundert. Bern 2003, S. 289-292. Alle Links in diesem Beitrag wurden am 26.03.2018 überprüft. 
1972 existierten sogar 92 Bibliotheken an der ganzen Universität, 45 davon in der medizinischen Fakultät. Erste Bestrebungen, die disperse Bibliothekslandschaft punktuell $\mathrm{zu}$ konzentrieren und Kleinbibliotheken $\mathrm{zu}$ fusionieren, setzten um 1970 ein: 1966 entstand die Universitätsspitalbibliothek, 1981 die Fachbereichsbibliothek Bühlplatz, 1988 die Bibliothek Erziehungswissenschaften und 1993 die Bibliothek Exakte Wissenschaften. Zudem führte die Informatik ab 1990 mit dem gemeinsamen Onlinekatalog die universitären Bibliotheken Berns zumindest virtuell näher zusammen. Mit dem Entscheid des Kantonsparlamentes von 1982, die räumliche Expansion der Universität nicht auf die grüne Wiese am Stadtrand zu verlagern, sondern durch eine Umnutzung der Schokoladenfabrik Tobler an der Länggassstrasse voranzutreiben, verzahnte sich die Hochschule noch stärker mit dem Quartier. 1993 zogen die meisten Institute der Philosophisch-historischen Fakultät sowie die Sozialwissenschaften und die Theologie mit rund einem Dutzend Bibliotheken in die Unitobler-Liegenschaft. Die StUB eröffnete gleichzeitig im überdachten Innenhof des Gebäudes die Basisbibliothek Unitobler. Die Universität erreichte damit zwar eine Reduktion der Bibliotheksstandorte, gleichzeitig verpasste sie die Chance, kleinere Bibliotheken organisatorisch zusammenzulegen und damit Synergien zu gewinnen und die Bibliotheken in diesem Gebäude als Gesamtes zu optimieren. So blieb ein strukturelles Problem bestehen: Die Existenz von vielen Institutsbibliotheken, die personell unterdotiert waren und die damit nicht den vollen bibliothekarischen Service anbieten konnten. $^{2}$

Ab 1990 gingen die Raumreserven der StUB an der Münstergasse zu Ende und bei einzelnen Institutsbibliotheken zeichneten sich ebenfalls Platzprobleme ab. Die StUB versuchte zuerst gemeinsam mit der Burgerbibliothek, ein unterirdisches Magazin am Aarehang zu realisieren. Dieses Projekt scheiterte jedoch in einer frühen Phase auf politischer Ebene, da die Kantonsbehörden und die Universität die nötigen Raumreserven an einem anderen Standort realisieren wollten und den Bau am Aarehang als zu teuer einschätzten. ${ }^{3}$

Der Kanton mietete als Zwischenlösung Lagerraum an der Hallerstrasse, wo 1997 bis 2013 wenig genutzte Bibliotheksbestände deponiert waren. Die Schweizerische Osteuropabibliothek befindet sich seit 1997 im selben Gebäude, sie übernahm die Betreuung dieses Aussenlagers.

2 Marianne Aeschbacher, Kilian Bühlmann, Roland de Loriol (Hrsg.): Die Bibliotheken der Universität Bern. Eine Übersicht. Festschrift für Werner Probst, Universitätsplaner von 1968-1997. Bern 1997; Minta/Nicolai/Thome, Stadt (wie Anm. 1), S. 81-95; Kilian Bühlmann: Stadt und Universität, eine Beziehungsgeschichte. In: UniPress 158 (2013), S. 5-8.

3 Der Bund, 6. Dezember 2001, S. 28; StUBsnase 3/2001, S. 3-6; Unilink, Januar 2002, S. 15. 


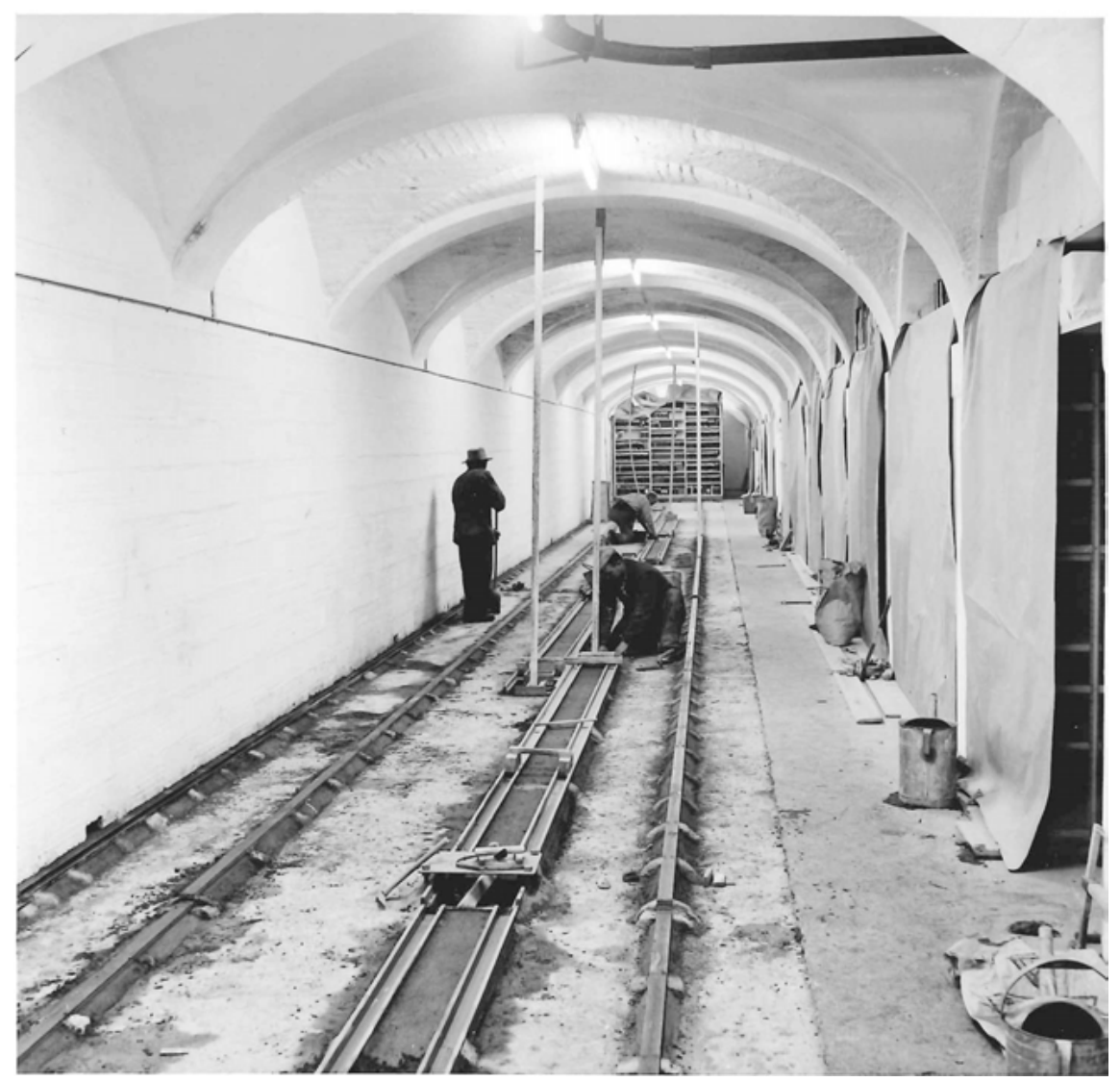

Abb. 2: Einbetonieren der Schienen für die erste Rollregalanlage in Bern, 1952 im Gewölbekeller der Stadt- und Universitätsbibliothek an der Münstergasse (@ Burgerbibliothek Bern)

\section{Bibliothek vonRoll}

Im Jahr 2000 erwarb der Kanton Bern zwei Drittel des früheren Industrieareals der Firma vonRoll an der Fabrikstrasse, um dort einen Campus für die wachsende Universität zu errichten. 2003 schrieb er einen Architekturwettbewerb aus, den die Architekten Lorenzo Giuliani und Christian Hönger aus Zürich gewannen. Zwei Jahre später setzte die Planung für dieses neue Hochschulzentrum ein. Die StUB und die Bibliothekskoordination der Universität waren von Anfang an daran beteiligt, da ein Teil des Raumprogramms ein Speichermagazin mit einer Kapazität von 80 Kilometern Rollregalen war, das Entlastung für die Lagerung der Printbestände aller Universitätsstandorte bringen sollte. Bis 2003 plante die Universität, naturwissen- 
schaftliche Fächer an diesem neuen Standort unterzubringen. 2004 fiel dann der Entscheid, den Neubau mit der Pädagogischen Hochschule und den sozial- und philosophisch-humanwissenschaftlichen Fächern der Universität zu belegen. Dazu gehörte eine grosse Fachbereichsbibliothek mit einer Fläche von 2'900 m². Einfacher wurde die Bibliotheksplanung, nachdem 2007 die StUB und die universitären Bibliotheken zur UB fusioniert wurden und eine ganze Reihe organisatorischer Schnittstellen wegfielen. In dieser Phase war auch Susanna Bliggenstorfer, als StUB- und UB-Direktorin von 2005 bis 2008, massgeblich an der Planung beteiligt.

Auf dem vonRoll-Areal wurde zuerst die frühere Weichenbauhalle von 1915 in ein Hörraumgebäude umgebaut. Anschliessend realisierte der Kanton 2010 bis 2013 den Neubau des Institutsgebäudes. Im dritten Untergeschoss dieser Liegenschaft entstanden das neue Speichermagazin der UB und darüber die Bibliothek vonRoll mit dem eindrücklichen zweigeschossigen Lesesaal und allen Betriebsräumlichkeiten. Dazu zählen die Büros der gut 60 Bibliotheksmitarbeitenden. ${ }^{4}$

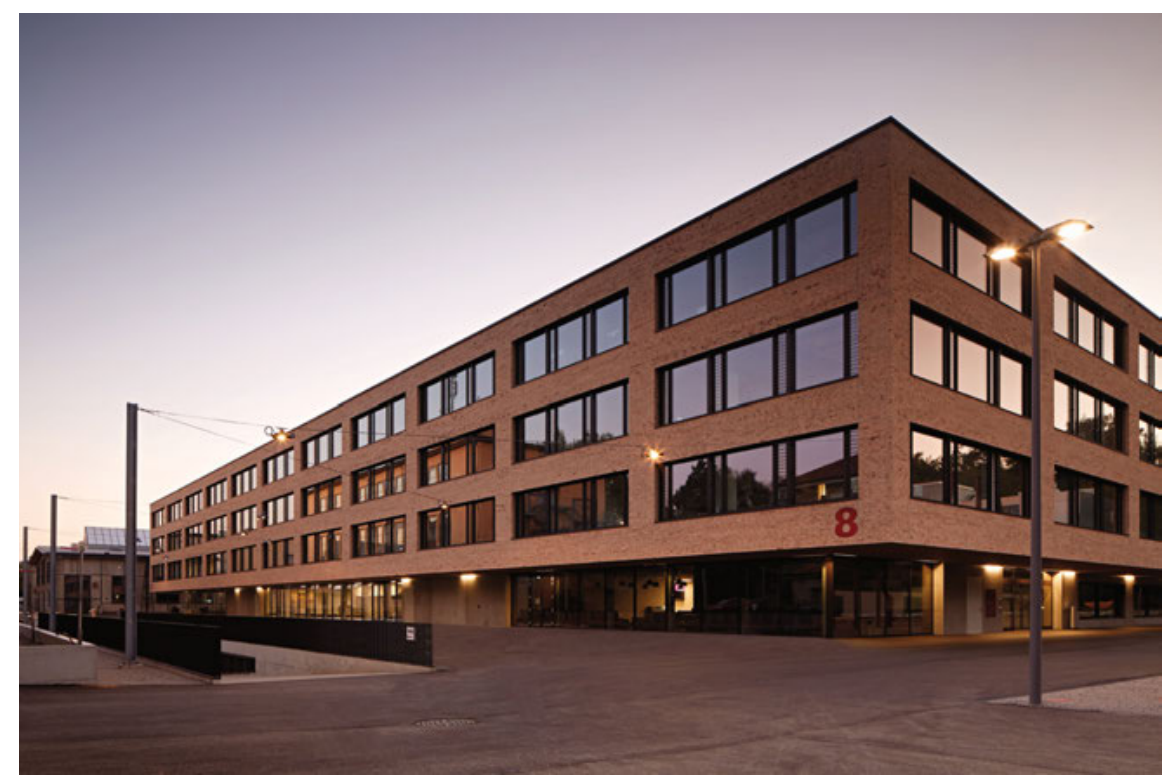

Abb. 3: Hochschulzentrum vonRoll (C Croci \& du Fresne Fotografie, September 2013 vor der Eröffnung), im Vordergrund der Neubau mit der Bibliothek in den Untergeschossen, im Hintergrund links die Weichenbauhalle, in die Hörräume eingebaut wurden

4 Amt für Grundstücke und Gebäude des Kantons Bern (Hrsg.): Hochschulzentrum vonRoll. Bern 2013; VonRoll-Areal Bern, Wettbewerb Primärsystem. In: hochparterre.wettbewerbe 4/2004, S. $1-$ 17; Der Bund, 30. Juni 2004, S. 23; 09. Juli 2004, S. 27; Minta/Nicolai/Thome, Stadt (wie Anm. 1), S. 223-225. 
Mit dem Speichermagazin entstand eine neue zentrale Einrichtung, die rund die Hälfte des Printbestandes der UB beherbergt. Die Gebäudestruktur mit ihren grossen Geschossflächen hatte zur Folge, dass als Bibliotheksmagazin kein Hochregallager, sondern ein Speicherkonzept mit einer Rollregalanlage zum Einsatz kam. Die Bau- und Einlagerungsplanung des Speichermagazins stellte die UB vor grosse Herausforderungen. Zum einen musste eine möglichst kompakte Anordnung der Rollregale gefunden werden, um die geplanten 80 Kilometer Regale in den Räumen unterzubringen. Zudem waren aus 17 Standorten insgesamt 60 Kilometer Bücher (2,2 Millionen Bände) zu zügeln. Davon stammten 35 Kilometer von der Zentralbibliothek (ehemals StUB) in der Münstergasse, 10 Kilometer aus dem Lager an der Hallerstrasse und weitere 10 Kilometer aus mehreren UnitoblerBibliotheken. Der Transfer war ein logistisches Grossprojekt, dessen Planung bereits Ende 2010 begonnen hatte. Basis für den Umzug war eine Einlagerungsliste für das Speichermagazin vonRoll: In einer Exceldatei wurde die Compactusanlage nach Regaleinheiten abgebildet. Die Datei hielt die Kapazität und die Einlagerungsmenge der Regaleinheiten, die Regaltiefe und die Anzahl der Tablare pro Block fest. Berücksichtigt wurden dabei einerseits die Formate, um den Platz optimal ausnützen zu können, und andererseits die Ausleihquoten. Je häufiger bestimmte Bestände ausgeliehen werden, desto näher stehen sie bei den Liftanlagen. So werden im Betrieb die Wege für das Magazinpersonal möglichst kurz gehalten. Die Umzugslisten hielten auch fest, welche Formate die Bücher haben, damit die Regale vor der Einlagerung mit der optimalen Zahl an Tablaren bestückt werden konnten. Der Umzug erfolgte mit Rollwagen, auf denen die Bücher in der richtigen Reihenfolge aufgeladen wurden. Während fünf Monaten verschob die Umzugsfirma rund 500 Laufmeter Bücher pro Arbeitstag. Nur mit dieser hohen Transportkapazität war das Projekt in einem überschaubaren Zeitraum zu bewältigen. 


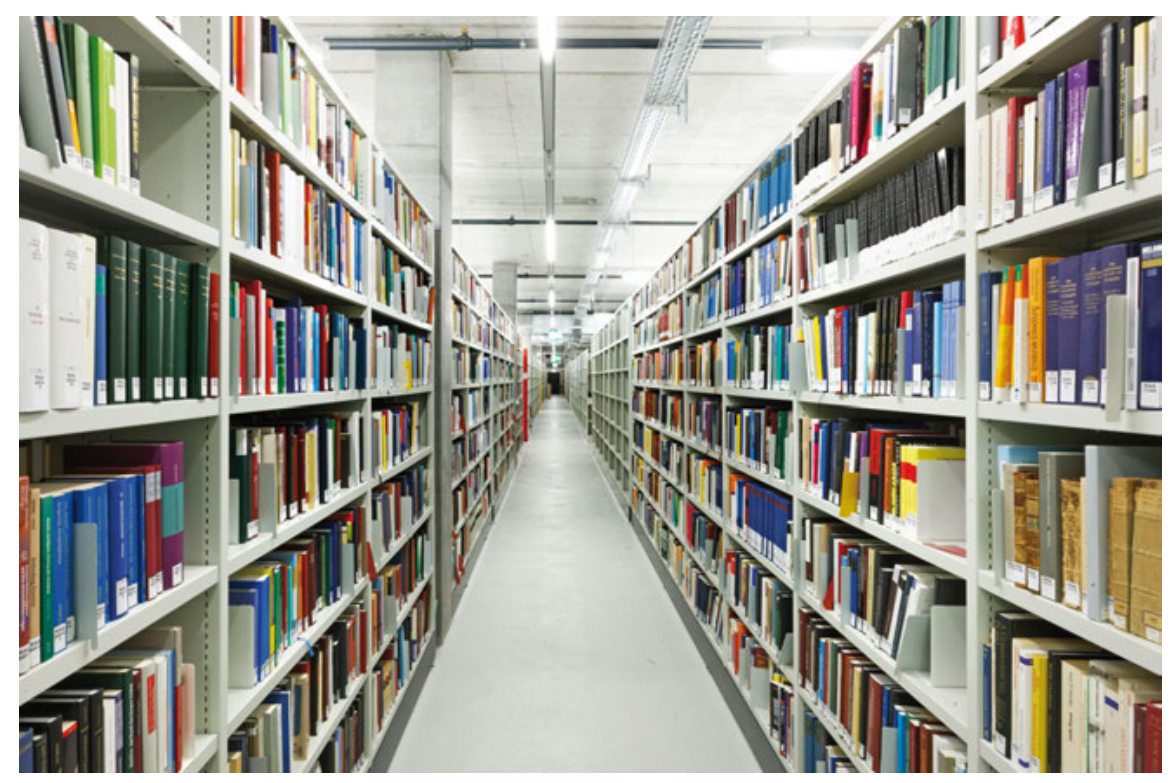

Abb. 4: Blick in den 94 Meter langen Hauptgang des UB-Speichermagazins vonRoll. In diesem Raum lagert rund die Hälfte der 4 Millionen Bände der UB Bern (C Iris Krebs 2014).

Mit dem Entscheid, das Speichermagazin am Stadtrand und trotzdem nahe bei der Universität zu realisieren, beseitigten die politischen Entscheidungsträger einen räumlichen Engpass der UB-Teilbibliotheken und erleichterten die Lagerlogistik, da Magazinräume an der Münstergasse, an der Haller- und an der Güterstrasse aufgehoben werden konnten. Zudem weist der vonRoll-Speicher der UB Zuwachsreserven von voraussichtlich 20 Jahren auf. Eine weitere Perspektive bietet eine unüberbaute Fläche auf dem Areal, wo in fernerer Zukunft weitere Neubauten des Kantons geplant sind. Darin ist eine Speichererweiterung denkbar. Dannzumal müssten auch weitere Optionen ins Auge gefasst werden, wie Bestände auszuscheiden oder ins Speichermagazin in Büron auszulagern, wie das 2016 andere Universitätsbibliotheken in der deutschen Schweiz gemacht haben. ${ }^{5}$

Der Bezug des vonRoll-Speichers der UB veränderte das räumliche Gefüge der UB grundlegend. Die Bibliothek vonRoll kam mit ihren rund 550 Publikumsarbeitsplätzen der grossen Nachfrage der Studierenden nach angenehmen Lernorten entgegen und entlastete andere Standorte. Zudem bietet die Bibliothek den

5 Siehe dazu die Beiträge von Daniel Tschirren und Ulrich Niederer in: b.i.t.online 18/6 (2015), S. 509-512 und in b.i.t.online 19/1 (2016), S. 74-77. Vgl. hierzu auch Beitrag „Der weite Blick“ von Ulrich Niederer im vorliegenden Band. 
PHBern-Studierenden ein qualitativ viel besseres Bibliotheksangebot als zuvor: Neben klassischen Lesesaalplätzen mit viel Tageslicht gehören 15 Gruppenarbeitsräume dazu, die gemeinsames Diskutieren und Lernen fördern. Die Bibliothek umfasst ausserdem ein Medienangebot von 140’000 Bänden, das in Freihandaufstellung präsentiert ist.

Die UB-Leitung entschied im Lauf der Planung, die Buchbinderei und die Fernleihe in der Bibliothek vonRoll zu konzentrieren. Zudem befinden sich hier das Servicezentrum Zeitschriften und eine Scanzentrale, die Zeitschriftenartikel und weitere Dokumente aus dem grossen Printbestand kopiert und als PDFDateien an die Kundschaft ausliefert. ${ }^{6}$

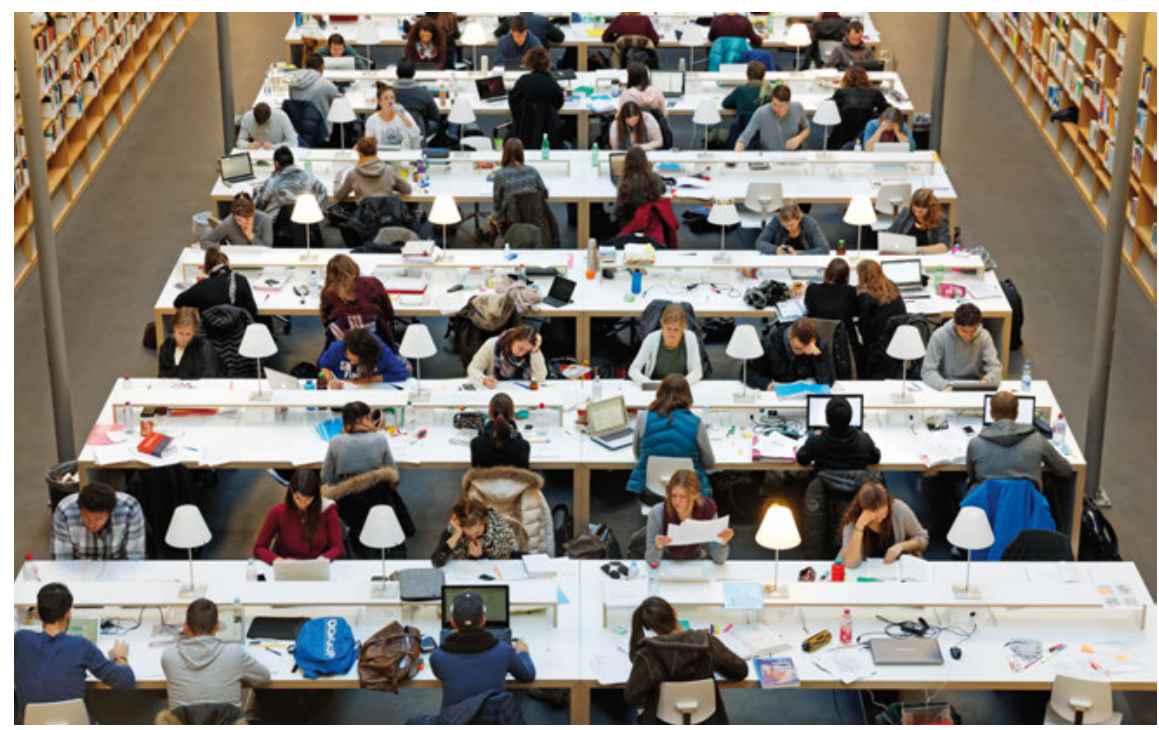

Abb. 5: Lesesaal der Bibliothek vonRoll 2014, ein viel genutzter Lernort (@ Iris Krebs)

\section{Bibliothek Münstergasse}

Die Liegenschaft Münstergasse 61/63 wurde 1968 bis 1974 umgebaut und um ein fünfgeschossiges unterirdisches Magazin erweitert. Etwa 90\% des Gebäudes nutzte nach diesem Umbau die Stadt- und Universitätsbibliothek, den Rest die Burgerbibliothek. Nach 40 Jahren war eine umfassende Sanierung des Gebäudes nötig geworden, die ab 2007 geplant wurde. Seit dem Umbau 2014 bis 2016 belegt

6 Universitätsbibliothek Bern: Jahresbericht 2013, S. 8f. 
die UB an diesem Standort noch rund 70\% respektive knapp 5’000 m² der Nutzfläche. Das Umbauprojekt gab den Anstoss, das Raum- und Dienstleistungskonzept der UB zu überdenken. Die Reduktion der verfügbaren Flächen zwang dazu, Schwerpunkte zu setzen. Mit dem neuen Konzept schafft die UB mehr Platz für Menschen und weniger für Bücher. Zudem wird das digitale Angebot stärker in den Vordergrund gerückt. So ist die Mitte 2016 neu eröffnete Bibliothek Münstergasse der UB ein öffentlich zugänglicher Lernort, der Standort der Historischen Bestände sowie ein Schwerpunkt der Medienbearbeitung.

In Abstimmung mit dem Standort vonRoll befindet sich an der Münstergasse nur ein geringer Anteil des Printbestandes. Dazu zählen einige tausend Referenzwerke und die neu aufgebaute Bernensia-Bibliothek in den Lesesälen. Die UB belegt nun noch ein Magazingeschoss, in dem die rund 250'000 historischen Bücher und Drucke mit Erscheinungsjahr vor 1800 und teilweise bis 1900 gelagert sind. Das Zentrum Historische Bestände (ZHB), eine Abteilung dieser Teilbibliothek, betreut den Bestand bibliothekarisch und konservatorisch. So befindet sich im Dachgeschoss des Westflügels das Restaurierungsatelier des ZHB, das auch Aufträge für die Burgerbibliothek im selben Haus ausführt. Im Sonderlesesaal können alle diese wertvollen Bestände konsultiert werden.

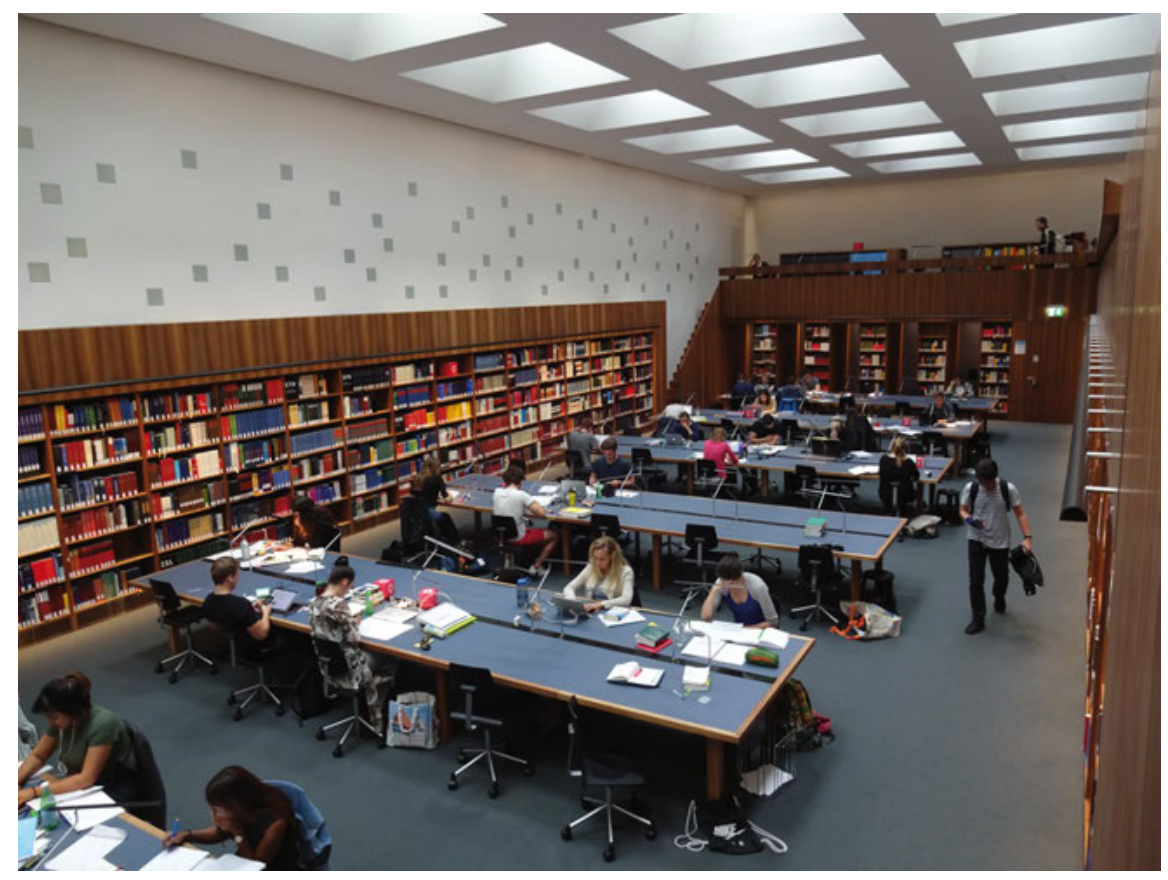

Abb. 6: Bibliothek Münstergasse, neuer Lesesaal im Untergeschoss (@ Christian Lüthi 2017) 
Rund die Hälfte der Flächen der Bibliothek Münstergasse sind für die Kundinnen und Kunden als Lernort nutzbar. Die Zahl der 330 verfügbaren Publikumsarbeitsplätze ist fast doppelt so hoch wie vor dem Umbau. Der Schultheissensaal im ersten Obergeschoss, der älteste profane Bibliothekssaal der Schweiz (gebaut 1787-1794), wurde sanft renoviert und dient wieder als repräsentativer historischer Lesesaal mit 60 Plätzen. Zusätzlich wurden die obersten beiden Geschosse des unterirdischen Magazinanbaus von $1974 \mathrm{zu}$ einem attraktiven neuen Lesesaal umgebaut. Unter der neu eingebauten Decke mit vielen Oberlichtfenstern befinden sich 92 Leseplätze, ergänzt durch 24 Einzelarbeitsplätze in seitlichen Nischen. Beide Säle haben sich seit der Wiedereröffnung als beliebte Räume für stilles Lernen bewährt.

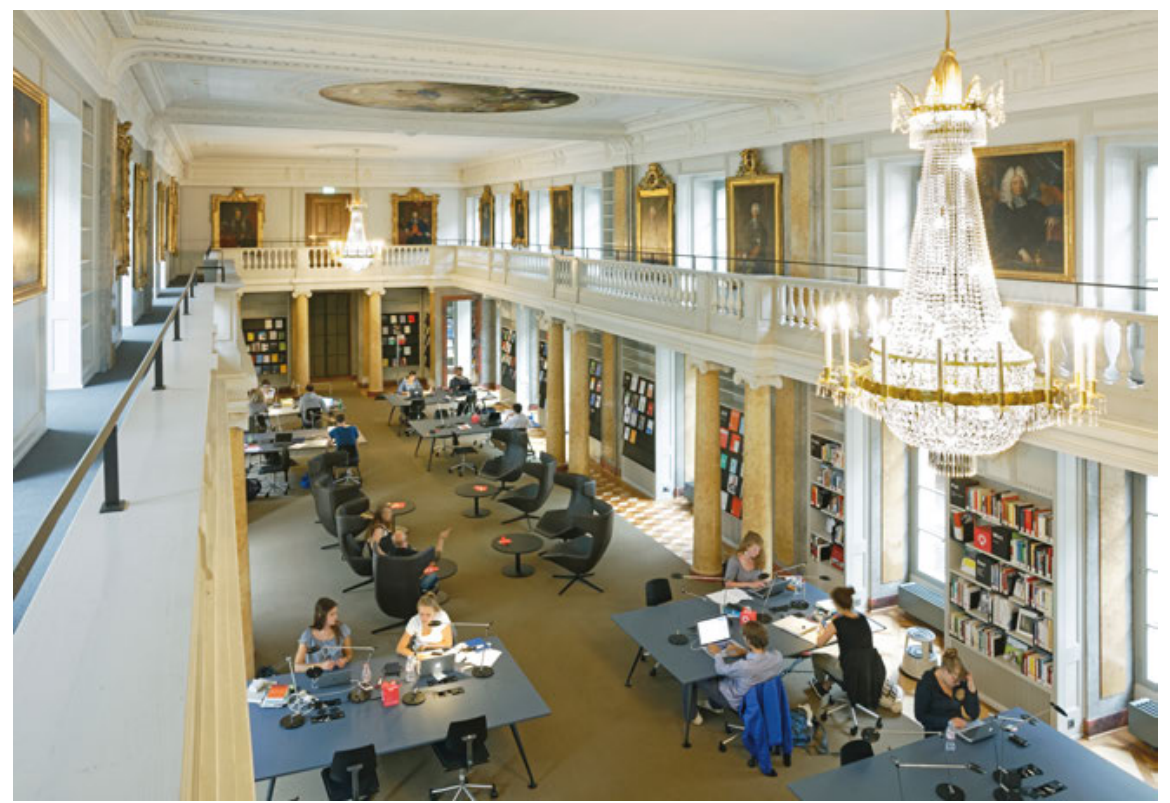

Abb. 7: Bibliothek Münstergasse, der renovierte Schultheissensaal, entstanden um 1790 (๑) Iris Krebs 2016)

$\mathrm{Zu}$ einem modernen Lernort gehören jedoch auch Räume für andere Lernformen. Dazu zählen die sechs neuen Gruppenarbeitsräume im Untergeschoss, die Leselounge mit Verpflegungsautomaten und einem Angebot an Tageszeitungen und Zeitschriften sowie der flexibel nutzbare Raum ,Information und Recherche‘. Für Schulungen steht ausserdem ein Kursraum mit PCs zur Verfügung; für Vorträge und kulturelle Veranstaltungen der Veranstaltungssaal mit 90 Plätzen. Beim 
grössten Andrang auf die Leseplätze während Prüfungszeiten lässt sich der Ausstellungsraum im historischen Gewölbekeller mit zusätzlichen Lesetischen möblieren. Sämtliche Publikumsräume sind mit WLAN ausgestattet: ein Muss, seit alle Studierenden neben einem Laptop ein Smartphone bei sich haben und auf mehreren Kanälen online sein möchten. Mit cUBe, das über lokale Sender (Beacons) und eine Handyapp standortbezogen Links und Zugang zum Online-Angebot der UB vermittelt, hat die UB erstmals versucht, ihre Lern- und Publikumsräume mit ihren virtuellen Räumen zu verknüpfen. ${ }^{7}$

Zwischen dem Lernen ist Entspannung und Verpflegung nötig. Im Erdgeschoss entlang der Laube zur Münstergasse hin gibt es seit der Neueröffnung ein Bibliothekscafé mit Bar, die LesBar. Der Raum verfügt neu über Fenster zur Gasse. Dank dieser Öffnung ist die Bibliothek von aussen sichtbarer. Die UBLeitung hat mit Unterstützung eines Gastroplaners eine Betreiberin für dieses Restaurant gesucht, das nicht über eine eigene Küche verfügt, aber trotzdem kleine Speisen, Snacks und Getränke im Angebot hat. Nach einem Jahr hat sich dieser Kleinbetrieb bestens etabliert und sich einen guten Ruf erarbeitet. ${ }^{8}$

Angesichts des neuen Profils dieses Standortes hat die UB den Namen von „Zentralbibliothek“, wie die Bibliothek ab 2007 hiess, in „Bibliothek Münstergasse“ geändert. Die Bibliothek ist einerseits nicht mehr der Ort für die Büros der UBLeitung und der zentralen Dienste und mit der Adresse im Namen lässt sich andererseits der Standort dieser Bibliothek besser nach aussen kommunizieren. Die neuen Räume machen die Bibliothek Münstergasse mit vollständig neuem Mobiliar und vor allem mit dem Konzept eines vielfältigen Lernraumes zu einem attraktiven Standort, der sich schnell als Schaufenster der Universität und der UB bei breiteren Kundenkreisen etabliert hat. ${ }^{9}$

7 Reto List, Isabelle Kirgus: Standortbasierte Online-Information vermitteln: cUBe, ein Projekt der Universitätsbibliothek Bern. In: o-bib 4/4 (2017), S. 63-70 (https://www.o-bib.de/article/ view/2017H4S63-70/6150); UB Bern: Jahresbericht 2016, S. 11; http://www.unibe.ch/universitaet/ dienstleistungen/universitaetsbibliothek/ub/cube/index_ger.html.

8 Vgl. www.lesbar.ch.

9 Burgerbibliothek Bern (Hrsg.): Durchblick. Umbau Archiv- und Bibliotheksgebäude Münstergasse. Bern 2016 (Passepartout); Isabelle Kirgus, Niklaus Landolt: Moderner Lernort in historischem Gebäude. Bibliothek Münstergasse als Teilbibliothek der UB Bern wiedereröffnet. Älteste öffentliche Bibliothek der Stadt Bern komplett saniert. In: BuB - Forum Bibliothek und Information 68 (2016), S. 562-567; Mahulena Svobodová (Hrsg.): New Library Buildings in Europe. Documentation 2014. Helsinki 2014, S. 152-166. 


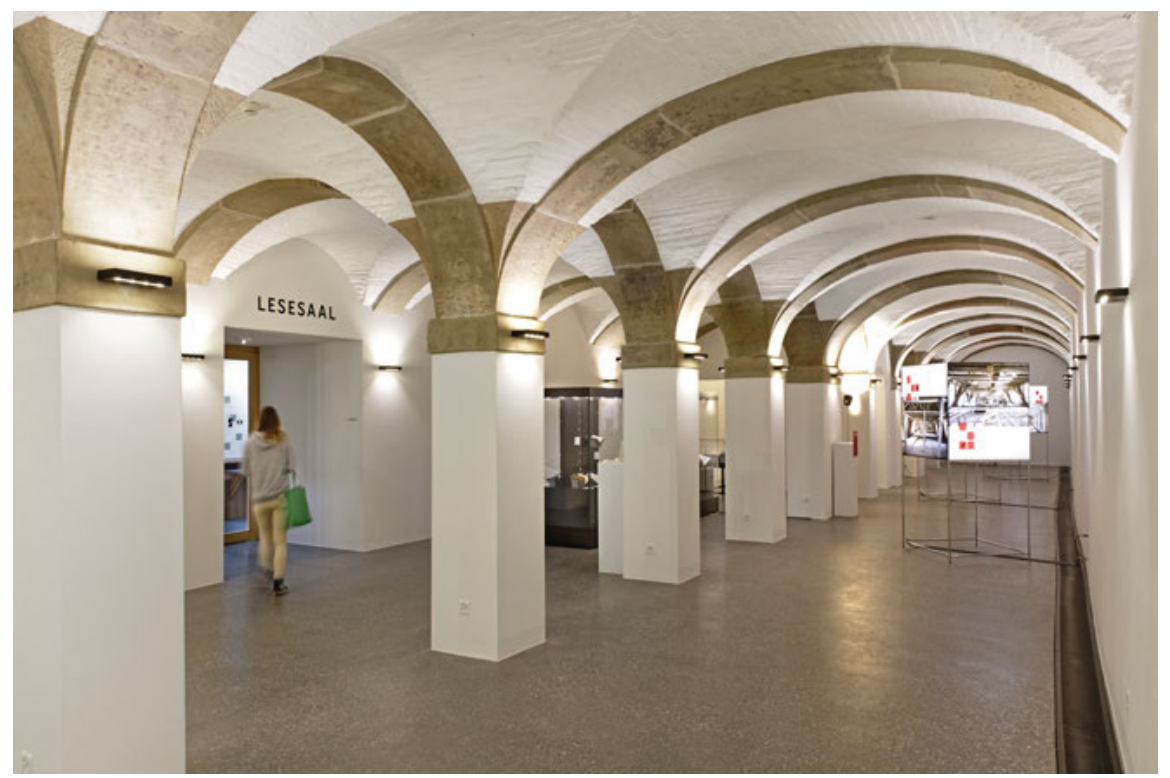

Abb. 8: Bibliothek Münstergasse, Gewölbekeller 2016. In diesem Raum befand sich bis 2013 die 1952 eingebaute Rollregalanlage, vgl. Abbildung 2 (৫ Iris Krebs)

\section{Das Konzept der Standortschwerpunkte}

Die beiden neuen Bibliotheken vonRoll und Münstergasse sind zwei der fünf Standortschwerpunkte der UB, also Teilbibliotheken mit den längsten Öffnungszeiten und dem umfassendsten Dienstleistungsangebot für die Kundinnen und Kunden. Der Ausbau der Standortschwerpunkte ist eines der strategischen Ziele der UB Bern. Mit diesem Konzept verfolgt sie das Ziel, ihre Kräfte zu bündeln. Neben den beiden genannten Bibliotheken zählen die Basisbibliothek Unitobler, die Juristische Bibliothek und die Fachbereichsbibliothek Bühlplatz zu den Standortschwerpunkten. Diese ,grossen Fünf‘ generieren mit 1'140'000 Besuchen pro Jahr (2016) rund 80\% der Kundenfrequenz der UB. Zudem bilden die Standortschwerpunkte mit vier weiteren Bibliotheken das Netz des Berner Kuriers, über den Bücher innerhalb der Stadt Bern zur Ausleihe an den gewünschten Abholort geliefert werden. Die rund 100'000 transportierten Bücher pro Jahr sind ein Beleg dafür, dass dieser Service einem grossen Bedürfnis entspricht.

Die UB Bern ist trotz ihrer immer noch recht heterogenen Raumstruktur als funktional einschichtige Bibliothek organisiert: Das Personal ist bei der UB angestellt und über die Hälfte der Erwerbskredite werden durch sie verwaltet. 
Zudem sind die E-Library sowie administrative und technische Dienstleistungen für den Betrieb räumlich zusammengelegt: Die UB-Leitung und die zentralen Dienste der UB haben ihre Büros seit 2015 an der Hochschulstrasse 6 im zentral gelegenen Verwaltungsgebäude der Universität. Ferner sind bereits weitere Raumprojekte der Universität aufgegleist, die es erlauben werden, kleine Bibliotheken zu grösseren Einheiten zusammenzufassen: 2018 wird die Bibliothek Mittelstrasse eröffnet, wo acht Bibliotheken zu einer Fachbereichsbibliothek mittlerer Grösse fusioniert werden. Zirka 2026 möchte die Hochschule zudem auf dem Areal des Inselspitals ein neues Ausbildungsgebäude beziehen, das auch Lernort mit Bibliotheksdienstleistungen sein wird. Mit demselben zeitlichen Planungshorizont wird auf dem Muesmattareal eine neue naturwissenschaftlich ausgerichtete Bibliothek die Fachbereichsbibliothek Bühlplatz ersetzen. Die Schwerpunktbildung ist also noch nicht abgeschlossen und bietet der UB Bern weitere interessante Bau- und Raumplanungsaufgaben. ${ }^{10}$

10 Der Bund, 06. Oktober 2016, S. 17. 
